\title{
Trapping and patterning of biological objects using photovoltaic tweezers
}

\author{
M. Jubera,${ }^{1}$ I. Elvira, ${ }^{1}$ A. García-Cabañes, ${ }^{1}$ J. L. Bella, ${ }^{2}$ and M. Carrascosa ${ }^{1}$ \\ ${ }^{1}$ Departamento de Física de Materiales, Universidad Autónoma de Madrid, Madrid 28049, Spain \\ ${ }^{2}$ Departamento de Biología, Universidad Autónoma de Madrid, Madrid 28049, Spain
}

(Received 1 October 2015; accepted 27 December 2015; published online 13 January 2016)

\begin{abstract}
Photovoltaic tweezers are a recently proposed technique for manipulation and patterning of microand nano-objects. It is based in the dielectrophoretic forces associated to the electric fields induced by illumination of certain ferroelectrics due to the bulk photovoltaic effect. The technique has been applied to the patterning of dielectric and metal micro- and nano-particles. In this work, we report the use of photovoltaic tweezers to pattern biological objects on $\mathrm{LiNbO}_{3}: \mathrm{Fe}$. Specifically, spores and pollen grains and their nanometric fragments have been trapped and patterned. 1D and 2D arrangements have been achieved by deposition in air or from a hexane suspension. The quality of patterns obtained with nanometric fragments is even better than previous results using photovoltaic tweezers with inorganic micro- and nano-particles. In fact, 1D patterns with a period of $2 \mu \mathrm{m}$, almost half of the minimum reported period achieved with photovoltaic tweezers, have been obtained with pollen fragments. C 2016 AIP Publishing LLC.
\end{abstract}

[http://dx.doi.org/10.1063/1.4939688]

Biological micro-patterning techniques provide a flexible tool for many areas of biological research, such as quantitative biochemical analysis, bio-sensing, and regenerative medicine. ${ }^{1-5}$ Among them, conventional micro-patterning techniques (e.g., photolithography and soft lithography) $)^{5,6}$ have undergone a significant adaptation to biological applications. However, they have not completely addressed the needs of constructing multi-object biological microarrays without requiring cleanroom access. Moreover, many of these technologies are rather sophisticated and expensive and are not available for most biological laboratories.

Dielectrophoretic (DEP) techniques based on the action of non-uniform electric fields on neutral matter ${ }^{7,8}$ are very suitable for bio-object manipulation (see Ref. 9 and references therein). They have a number of advantages, such as easy operation and high efficiency. However, they need voltage suppliers and micro-electrodes that have to be usually fabricated deposited in a substrate. In that case, they need again the use of a lithographic technique that must be sophisticated if small inter-electrode distance and high electric fields are required. To overcome this problem, an interesting optoelectronic DEP approach was proposed that uses light images to generate the electric fields in a photoconductor material. ${ }^{10,11}$ It avoids the need of micro-electrodes but still uses voltage suppliers.

Recently, an alternative optoelectronic technique for micro- and nano-particle trapping and patterning, the socalled photovoltaic (PV) tweezers, ${ }^{12}$ has been proposed and demonstrated. It relies in the bulk PV effect presented by doped $\mathrm{LiNbO}_{3}$ crystals that allows the generation of very high internal electric fields $\left(\sim 10^{4}-10^{5} \mathrm{~V} / \mathrm{cm}\right)$ induced by the light illumination. The technique, at variance with other DEP methods, is free of voltage suppliers and real electrodes. Besides, at difference with standard optical tweezers, it operates with moderate or low light intensity levels $\left(\sim \mathrm{mW} / \mathrm{cm}^{2}\right)$ and can act simultaneously on many particles. ${ }^{12}$ The induced spatial electric field distribution is closely correlated with the light illumination pattern and extends to the proximity of the crystal surface acting on the existent objects. It can trap either charged or neutral micro- and nano-particles, via electrophoretic or DEP forces, respectively. ${ }^{7,8}$ The periodicity of the light-induced electric field distribution (i.e., the distance between "virtual electrodes") can reach a value as small as $3.5 \mu \mathrm{m}$ as recently reported. ${ }^{13}$ In principle, substantially smaller periods, even in the sub-micrometric range, should be reachable, but further optimization of the technique would be needed. Although a rather high number of works have been published describing the physical properties and possible technological applications of PV tweezers, ${ }^{14-19}$ very few of them are in the field of biology. Only relevant effects appearing in the interaction of the PV field with microorganisms, ${ }^{20}$ and more recently, with cancer cells ${ }^{21}$ and Escherichia coli bacteria $^{22}$ have been reported. In fact, although it is expected that the technique can be used as a relatively simple and low cost method for massive micro-patterning of biological objects, the demonstration of these specific capabilities is still lacking.

In this work, we apply this rather simple technique to pattern different types of bio-objects, spores and pollen, and their fragments. These bio-particles are of particular practical importance either in food technologies or in medicine in connection with allergic respiratory diseases. Note that pollen fragments seem to have even a higher allergenic activity. ${ }^{23,24}$ The bio-particle deposition will be carried out in air or from a liquid suspension. 1D and 2D periodic patterns, with periods in the range between $2 \mu \mathrm{m}$ and $700 \mu \mathrm{m}$, will be obtained. The results will be discussed in comparison with previous results for inorganic particles. In particular, the smallest period value of $2 \mu \mathrm{m}$, achieved in this work, is a record for this kind of tweezers, lower than the minimum period obtained so far. ${ }^{13}$ The results will show the very good capabilities of PV tweezers to trap and pattern bio-objects and open the door to a wide number of applications in biotechnology.

The patterning procedure includes two steps: (i) generation of the PV field pattern by illuminating the PV material 

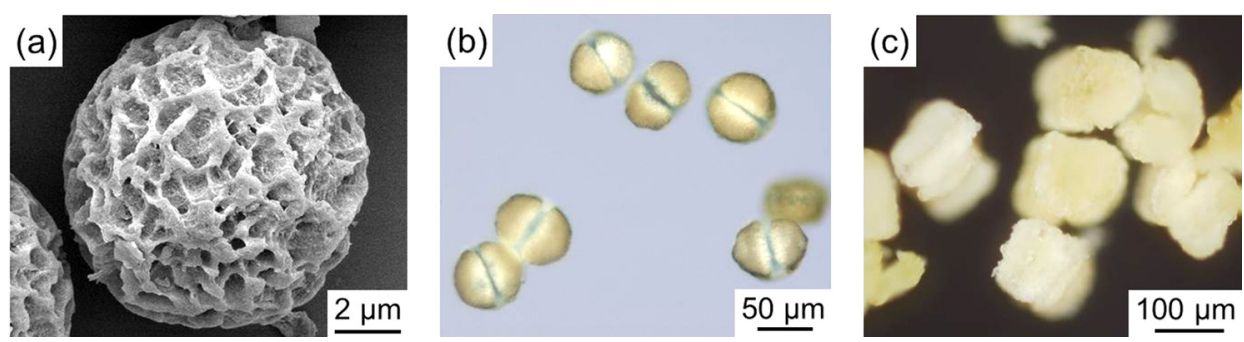

FIG. 1. (a) SEM image of a spore of a puffball mushroom (Lycoperdon). Optical microscope images of (b) Himalayan cedar (Cedrus deodar) and (c) Mimosa (Acacia dealbata) pollen grains.

$\left(\mathrm{LiNbO}_{3}: \mathrm{Fe}\right.$ in our case) with a suitable light pattern, and (ii) bio-object deposition by approaching them to the substrate in air or suspended in a liquid.

The PV effect is a consequence of the noncentrosymmetric crystal lattice of the PV material. Visible illumination induces a directional excitation of electrons ${ }^{25}$ from the $\mathrm{Fe}^{2+}$ donor impurities along the ferroelectric trigonal (poling) axis. Then, electrons are trapped in available $\mathrm{Fe}^{3+}$ acceptors. As a consequence, charge separation occurs, generating a space-charge field inside the $\mathrm{LiNbO}_{3}: \mathrm{Fe}$ crystal that is closely correlated with the light pattern and extends outside its surface. ${ }^{12,26}$

Neutral particles in the proximity of the crystal polarize and undergo the so called DEP force. For spherical isotropic particles and assuming the Rayleigh approximation, a simple expression for the DEP force applies ${ }^{12}$

$$
\vec{f}=-\alpha \vec{\nabla}\left(\vec{E}_{P V}^{2}\right)
$$

where $\alpha$ is the particle polarizability that can be written as

$$
\alpha=2 \pi r^{3} \varepsilon_{m} \frac{\varepsilon_{p}-\varepsilon_{m}}{\varepsilon_{p}+2 \varepsilon_{m}}
$$

being $r$ the radius of the particle and $\varepsilon_{p}$ and $\varepsilon_{m}$ the relative dielectric permittivities of the particle and the medium surrounding the PV substrate, respectively. Note that the magnitude of the DEP force linearly depends with the volume of the bio-object and the difference between the dielectric permittivities of the particle and the deposition medium. In this work we use two deposition media that are appropriate to trap and pattern pollen and spores, air $\left(\varepsilon_{m}=1\right)$ and hexane $\left(\varepsilon_{m}=1.9\right)$. On the other hand, as discussed elsewhere, ${ }^{12,13}$ the DEP force decays exponentially along the direction normal to the surface. For sinusoidal light patterns, the decay spatial constant is $\sim \Lambda / 18, \Lambda$ being the light pattern period.

In order to check the viability to pattern bio-objects with PV tweezers we have started the study addressing experiments using micrometric spores from a puffball mushroom (Lycoperdon) and two types of pollen, from Himalayan cedar (Cedrus deodara) and from mimosa (Acacia dealbata). Figure 1 shows microscopic images of these species. Spores are spherical with a diameter $d=10 \mu \mathrm{m}$, and pollen grains are also nearly spherical but with larger diameters, $d \sim 60$ and $120 \mu \mathrm{m}$ for Himalayan cedar and mimosa, respectively. A second set of experiments use nanometric fragments of these bio-objects obtained by sonication of a hexane suspension.

As PV substrate we have used $\mathrm{LiNbO}_{3}$ crystals highly doped with iron as in most experiments with PV tweezers. Two crystal orientations, $x$ - and $z$-cuts, i.e., samples with the polar axis ( $c$-axis) parallel or normal to the surface, respectively, are used. A frequency doubled Nd:YAG operating at $532 \mathrm{~nm}$ is utilized to illuminate the substrate. 1D patterns are mainly obtained under periodic illumination of $x$-cut substrates obtained from the interference of two light beams ${ }^{27}$ (see Fig. 2(a)). However, in a few 1D experiments with large period, the light periodic fringe pattern is provided by a spatial light modulator (SLM). In turn, 2D patterns are fabricated illuminating $z$-cut substrates with a suitable $2 \mathrm{D}$ light distribution provided by the SLM. This latter crystal orientation, recently proposed, is a key point to obtain 2D patterns that accurately reproduce the light pattern. ${ }^{19,28,29}$ The light illumination set-up for experiments using the SLM is shown in Fig. 2(b).

Let us start showing the results using micrometric spores $(d \sim 10 \mu \mathrm{m})$. A $1 \mathrm{D}$ patterning experiment was carried out in a $x$-cut substrate and under sinusoidal illumination $(\Lambda=35 \mu \mathrm{m})$. The spores were deposited immersing the substrate in a hexane suspension. Fig. 3(a) shows an image of the observed spore pattern with the same periodicity of light whereas Fig. 3(b) shows the result of a control experiment in which the substrate has been immersed in the hexane suspension without any previous illumination. In Fig. 3(a) it can be clearly seen a 1D pattern formed by lines of spores perpendicular to the polar $c$-axis, whereas, without PV fields (Fig. 3(b)), only some spores remain on the surface but located at random. It can be remarked that the ratio between the light period $\Lambda$ and the particle diameter $d$, $\Lambda / d \sim 4$, is smaller than the typical values obtained in previous experiments (see for instance Ref. 13). In other words, for a given light period $\Lambda$, we are able to trap objects

(a)

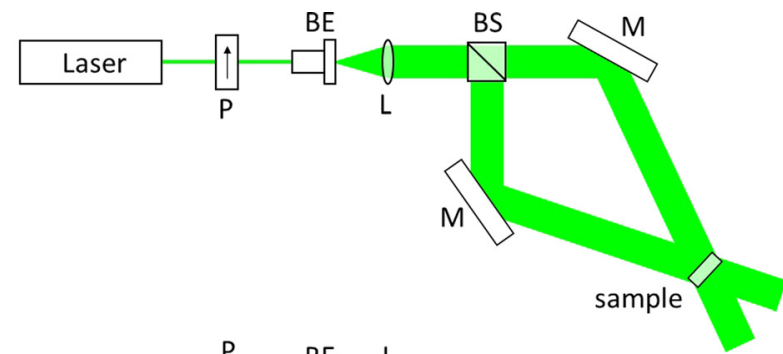

(b)

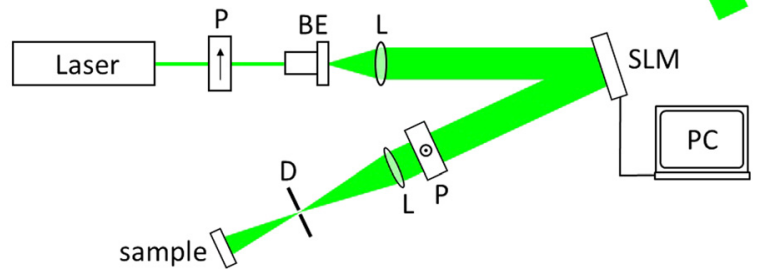

FIG. 2. Experimental set-ups used to obtain the different light illumination patterns: (a) Holographic configuration for 1D sinusoidal illumination. (b) Spatial Light Modulator (SLM) for 2D arbitrary illumination. BE, beam expander; BS, beam splitter; D, diaphragm; L, lens; M, mirror; P, polarizer. 

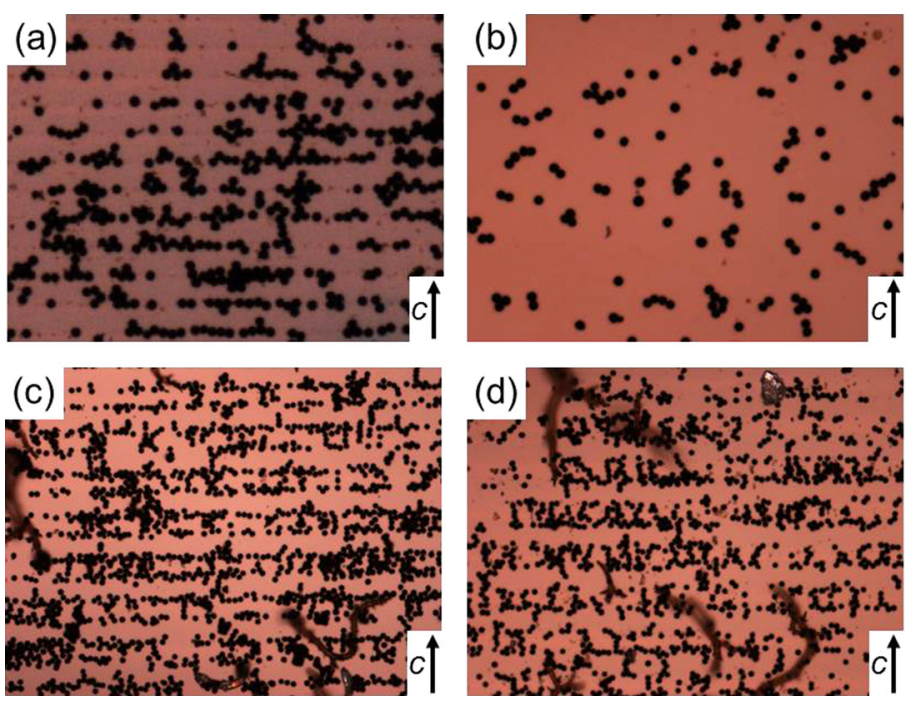
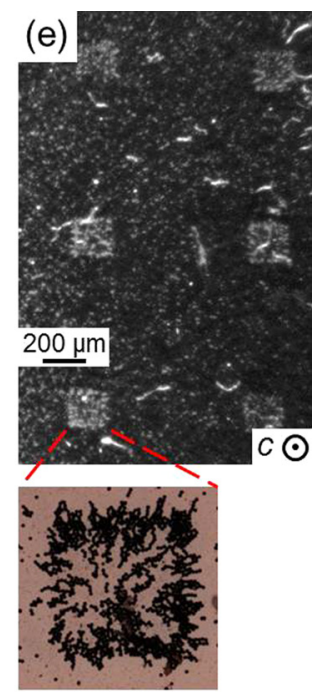

FIG. 3. (a) Microphotographs of a pattern $(\Lambda=35 \mu \mathrm{m})$ obtained after sinusoidal illumination and deposition of Lycoperdon spores from a hexane suspension. (b) Control experiment with a non-illuminated substrate, i.e., without PV fields. (c) and (d) Microphotographs of spore patterns $(\Lambda=85 \mu \mathrm{m})$ obtained under sinusoidal illumination with two deposition media, hexane and air, respectively. (e) Photograph of a 2D pattern with six square traps for spores deposited in hexane. A magnification (microphotograph) of one of the squares to see the individual spores is shown on the bottom. (spores) with a size larger than in usual inorganic particle experiments. Some patterning experiments have been conducted with deposition in air, spreading the spores on the previously illuminated substrate. For comparison purposes Figs. 3(c) and 3(d) show the patterns obtained by deposition in air and from a hexane suspension, respectively, under sinusoidal illumination with a light pattern period $\Lambda=85 \mu \mathrm{m}$. It is clearly seen the high similarity between both patterns showing that deposition in air is also effective. Finally, 2D patterning has been also attempted using $z$-cut crystals and illumination through an SLM. In Fig. 3(e) immobilization of spores in an array of square traps is shown. A magnification of one of the squares is also presented in the bottom of the figure. There, it can be seen that most spores are arranged forming chains. These chains are typical of the action of DEP forces and stems from the dipole-dipole interaction. ${ }^{8}$ The chains tend to align along the horizontal component of the PV electric field at the substrate surface that is roughly radial in each illuminated square. Some DEP chains can be also seen in Figs. 3(c) and 3(d). All these results are a clear demonstration of the capabilities of PV tweezers to induce micro-patterning of biological objects.

These capabilities are further illustrated with the second set of experiments carried out using the mimosa pollen grains. In this case, they have been placed in air on the previously illuminated sample covering it homogeneously. Afterwards, those not attached to the surface were removed by blowing. Due to the relatively large size of these pollen grains, only more macroscopic patterns (with periods ranging between $0.1-1 \mathrm{~mm}$ ) have been obtained. In Fig. 4 we show two representative results. The first one is obtained using a periodic 1D light pattern provided by the SLM. Fig. 4(a) shows a global view of the 1D pattern and a magnification of a smaller region. It can be clearly seen that the pollen grains organize in lines perpendicular to the polar axis. The second result, Fig. 4(b), corresponds to a $z$-cut crystal illuminated by an array of squares. The grains are trapped and organized along the illuminated regions as it can be observed in the image.

So far, we have patterned micrometric objects but a further step is to check the possibility of trapping and structuring nanometric biological particles. Note that DEP forces scale with the particle volume (see Eq. (2)) and will be much smaller. To this end, we have obtained by sonication pollen and spores fragments whose sizes result substantially smaller than $1 \mu \mathrm{m}$. The pollen fragments (Himalayan cedar) are shown in Fig. 5(a) where it can be seen that typical diameters are in the range of $50-150 \mathrm{~nm}$. High quality $1 \mathrm{D}$ and $2 \mathrm{D}$ patterns of these pollen components are obtained as shown in the microscopic images of Figs. 5(b) and 5(c), respectively. It is remarkable the good definition of the lines and squares similar or even better than those recently reported ${ }^{19,28}$ using micro- and nano-particles. In fact, at difference with previous work, the bio-particles fully fill the deposition regions.

Due to the good results obtained, we addressed a set of experiments to reduce the period of $1 \mathrm{D}$ patterns, i.e., to investigate the maximum resolution of the patterning technique. In Figs. 5(d)-5(f) we show microscopic images of the obtained results for periods of $20 \mu \mathrm{m}, 8 \mu \mathrm{m}$, and $2 \mu \mathrm{m}$. It is observed the good quality of the patterns for $20 \mu \mathrm{m}$ and $8 \mu \mathrm{m}$, still with high particle density. For the smallest period the definition is not so good but the pattern is easily
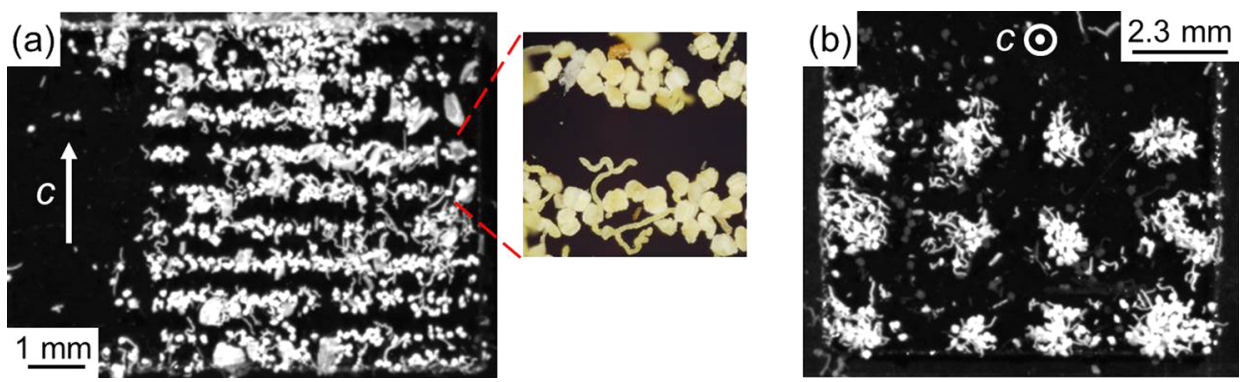

FIG. 4. (a) Photograph of a 1D pattern of mimosa pollen grains and a magnification (microphotograph) of a smaller region. (b) Photograph of a $2 \mathrm{D}$ pattern of the same pollen grains. 

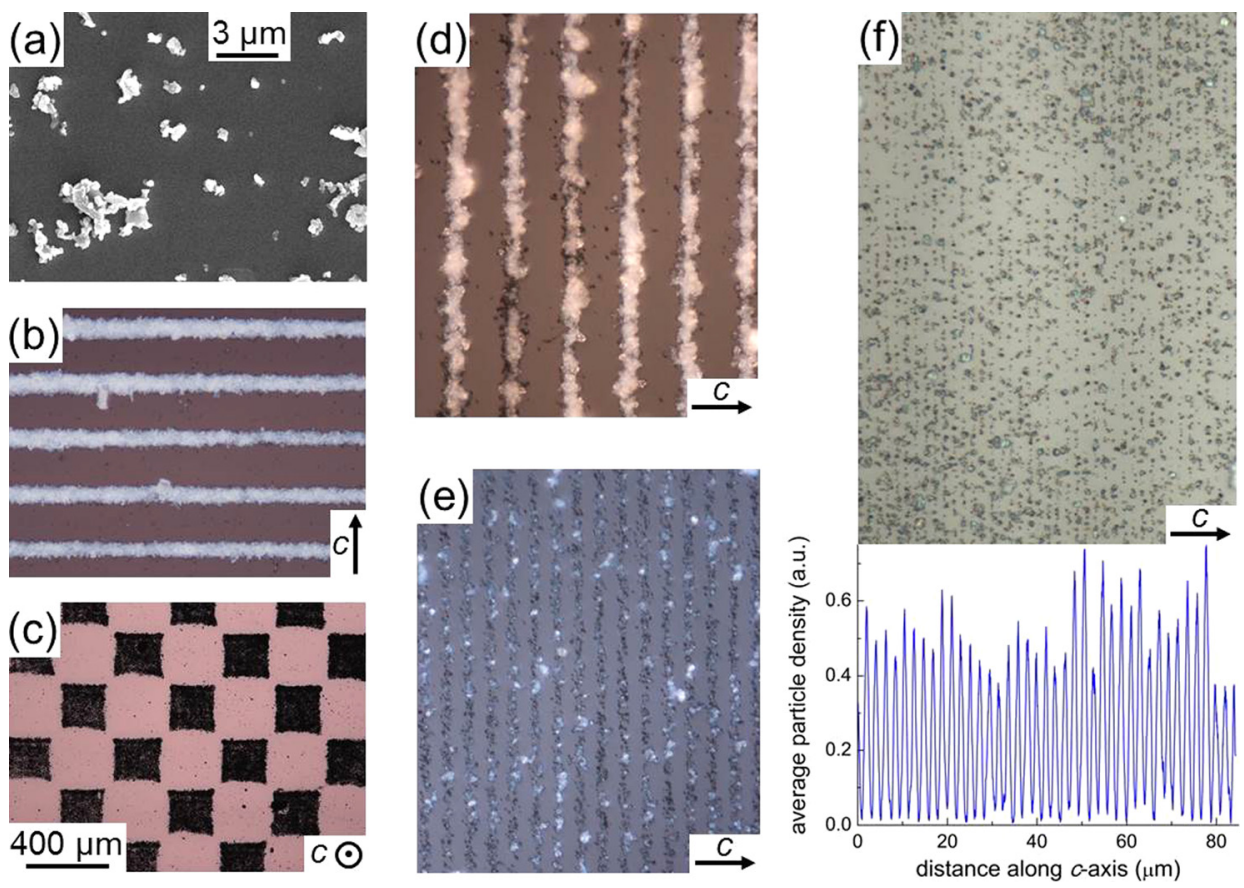

FIG. 5. (a) SEM image of Himalayan cedar pollen fragments. (b) Microscope image of a $65 \mu \mathrm{m}$ period pattern of these pollen fragments obtained with sinusoidal illumination of the same spatial period. (c) Microscope image of a 2D pattern of the same pollen fragments obtained after illumination with a mosaic of squares with $200 \mu \mathrm{m}$ side. Microscope images of periodic patterns with decreasing periods: (d) $\Lambda=20 \mu \mathrm{m}$, (e) $\Lambda=8 \mu \mathrm{m}$, and (f) $\Lambda=2 \mu \mathrm{m}$. In the bottom of the last one (f), the corresponding average particle density profile along the $c$-axis direction is also shown. observed. To better appreciate the periodic structure for the last case $(\Lambda=2 \mu \mathrm{m})$, a profile along the $c$-axis of the average density of particles in the direction parallel to the fringes (i.e., normal to the $c$-axis) is shown in the bottom of Fig. 5(f). It can be clearly seen the periodicity of the trapped particle pattern. It is remarkable that this periodicity is a record for this kind of tweezers since the minimum reported period, obtained with metal nanoparticles, is $3.5 \mu \mathrm{m} .{ }^{13}$ Then, these results are an additional demonstration of the potential of this kind of tweezers that can also trap and organize nanometric bio-objects, opening the door to a wider number of applications in biotechnology.

Finally, nanometric fragments of spores, even smaller than those of pollen grains, were also used, as shown in the SEM image of Fig. 6(a). They can be also patterned with a similar quality to that of pollen fragment patterns. Fig. 6(b) presents a spore fragment pattern $(\Lambda=15 \mu \mathrm{m})$ with welldefined and thin lines of about $1 \mu \mathrm{m}$ width, as it is clearly seen in the SEM image magnification of one line shown on the right.

The efficient patterning of nanometric bio-particles is a remarkable result of this work. The ability of PV tweezers to trap nano-objects, already found with inorganic nanoparticles, might be related with the limited vertical range of DEP forces (about $\Lambda / 18$ ). ${ }^{13}$ Hence, when nanoparticles approach the PV sample the whole particle undergoes high
DEP forces whereas micro-particles could be partially outside the DEP force spatial range.

In summary, the application of PV tweezers for efficient trapping and patterning of various types of bio-objects (spores, pollen, and their fragments) has been clearly demonstrated. The patterning technique is based on DEP forces but does not need voltage suppliers or electrodes. Moreover, the quality of the patterns obtained with nanometric pollen fragments is similar or even better than that of the patterns obtained using the same technique with inorganic nanoparticles. Specifically, the spatial resolution of $1 \mathrm{D}$ patterns is remarkably high, achieving periodicities of $2 \mu \mathrm{m}$, lower than the best reported ones, and the definition of 2D patterns is also very good compared with previous results in the literature. The results of trapping and patterning may open the possibility of a variety of applications such as pollen analysis and sensing where other electro-kinetic methods are currently explored. ${ }^{30}$ As another example, this methodology may also potentially serve to discriminate among different kinds of pollen and spores, or other bio-objects with interest for industry, science, or technology. Moreover, since deposition is usually carried out from a liquid suspension, the technique could easily be applied in bio-microfluidics. A rapid development of these kind and other biological applications of the technique is expected in the near future.
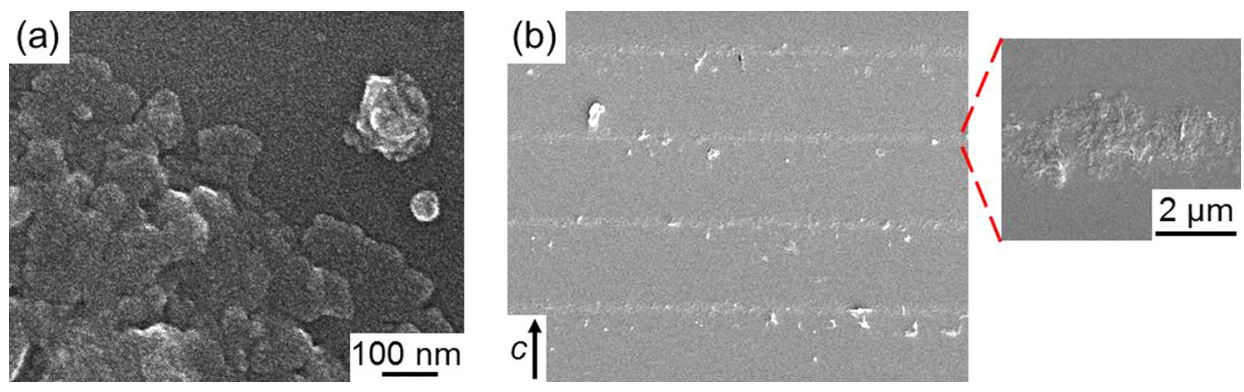

FIG. 6. SEM images of (a) Lycoperdon spore fragments and (b) a $15 \mu \mathrm{m}$ period pattern of these spore fragments obtained with sinusoidal illumination of the same spatial period, together with a magnification of a line of this pattern. 
This work was supported by Spanish projects MAT2011-28379-C03 and MAT2014-57704-C03. We thank Dr. Victor Jiménez-Rico (UCM) who kindly provided us the Himalayan cedar pollen grains.

${ }^{1}$ C. S. Chen, M. Mrksich, S. Huang, G. M. Whitesides, and D. E. Ingber, Science 276, 1425 (1997).

${ }^{2}$ M. J. Heller, Annu. Rev. Biomed. Eng. 4, 129 (2002).

${ }^{3}$ J. Nakanishi, T. Takarada, K. Yamaguchi, and M. Maeda, Anal. Sci. 24, 67 (2008).

${ }^{4}$ A. Ranga, S. Gobaa, Y. Okawa, K. Mosiewicz, A. Negro, and M. P. Lutolf, Nat. Commun. 5, 4324 (2014).

${ }^{5}$ E. D'Arcangelo and A. P. McGuigan, BioTechniques 58, 13 (2015).

${ }^{6}$ Y. N. Xia and G. M. Whitesides, Annu. Rev. Mater. Sci. 28, 153 (1998).

${ }^{7} \mathrm{H}$. Pohl, Dielectrophoresis: The Behavior of Neutral Matter in Nonuniform Electric Fields (Cambridge University Press, 1978).

${ }^{8}$ T. B. Jones, Electromechanics of Particles (Cambridge University Press, 1995).

${ }^{9}$ Ch. Qian, H. Huang, L. Chen, X. Li, Z. Ge, T. Chen, Z. Yang, and L. Sun, Int. J. Mol. Sci. 15, 18281 (2014).

${ }^{10}$ P. Y. Chiou, A. T. Ohta, and M. C. Wu, Nature 436, 370 (2005).

${ }^{11}$ A. Jamshidi, P. J. Pauzauskie, P. J. Schuck, A. T. Ohta, P. Y. Chiou, J. Chou, P. D. Yang, and M. C. Wu, Nat. Photonics 2, 86 (2008).

${ }^{12}$ J. Villarroel, H. Burgos, A. García-Cabañes, M. Carrascosa, A. BlázquezCastro, and F. Agulló-López, Opt. Express 19, 24320 (2011).

${ }^{13}$ J. Matarrubia, A. García-Cabañes, J. L. Plaza, F. Agulló-López, and M. Carrascosa, J. Phys. D: Appl. Phys. 47, 265101 (2014).

${ }^{14}$ H. A. Eggert, F. Y. Kuhnert, K. Buse, J. R. Adleman, and D. Psaltis, App. Phys. Lett. 90, 241909 (2007).

${ }^{15}$ X. Zhang, J. Wang, B. Tang, X. Tan, R. A. Rupp, L. Pan, Y. Kong, Q. Sun, and J. Xu, Opt. Express 17, 9981 (2009).
${ }^{16}$ M. Esseling, F. Holtmann, M. Woerdemann, and C. Denz, Opt. Express 18, 17404 (2010).

${ }^{17}$ L. Miccio, P. Memmolo, S. Grilli, and P. Ferraro, Lab Chip 12, 4449 (2012).

${ }^{18}$ M. Jubera, A. García-Cabañes, J. Olivares, A. Alcazar, and M. Carrascosa, Opt. Lett. 39, 649 (2014).

${ }^{19}$ M. Esseling, A. Zaltron, C. Sada, and C. Denz, Appl. Phys. Lett. 103, 061115 (2013).

${ }^{20}$ N. Kukhtarev, T. Kukhtareva, J. Jones, E. R. Ward, S. Sarkisov, and M. Curley, Proc. SPIE 4744, 272 (2002).

${ }^{21}$ A. Blázquez-Castro, J. C. Stockert, B. López-Arias, A. Juarranz, F. Agulló-López, A. García-Cabañes, and M. Carrascosa, Photochem. Photobiol. Sci. 10, 956 (2011).

${ }^{22}$ L. Miccio, V. Marchesano, M. Mugnano, S. Grilli, and P. Ferraro, Opt. Laser Eng. 76, 34 (2016).

${ }^{23}$ R. B. Knox, Clin. Exp. Allergy 23, 354 (1993).

${ }^{24}$ G. D'Amato, L. Cecchi, S. Bonini, C. Nunes, I. Annesi-Maesano, H. Behrendt, G. Liccardi, T. Popov, and P. van Cauwenberge, Allergy 62, 976 (2007).

${ }^{25}$ B. Sturmann and V. Fridkin, Photovoltaic and Photorefractive Effects in Noncentosymetric Materials (Gordon and Breach Science Publishers, Philadelphia, 1992).

${ }^{26}$ F. Agulló-López, G. F. Calvo, and M. Carrascosa, in Photorefractive Materials and Their Application, edited by P. Günter and J. P. Huignard (Springer, New York, 2006), Chap. 3, p. 43.

${ }^{27}$ H. Burgos, M. Jubera, J. Villarroel, A. García-Cabañes, F. Agulló-López, and M. Carrascosa, Opt. Mater. 35, 1700 (2013).

${ }^{28}$ J. F. Muñoz-Martínez, I. Elvira, M. Jubera, A. García-Cabañes, J. Bruno Ramiro, C. Arregui, and M. Carrascosa, Opt. Mater. Express 5, 1137 (2015).

${ }^{29}$ C. Arregui, J. B. Ramiro, A. Alcázar, A. Mendez, J. F. Muñoz-Martínez, and M. Carrascosa, J. Eur. Opt. Soc. 10, 15026 (2015).

${ }^{30}$ C. Kawaguchi, T. Noda, M. Tsutsui, M. Taniguchi, S. Kawano, and T. Kawai, J. Phys.: Condens. Matter 24, 164202 (2012). 